*For correspondence:

azembrzycki@salk.edu

${ }^{\dagger}$ These authors contributed equally to this work

Present address: ${ }^{\ddagger}$ Biosciences Department, Minnesota State University, Moorhead, United States; ${ }^{\S}$ University Cancer Center Hamburg, University Medical Center, Hamburg, Germany; "MRC Centre for Developmental Neurobiology, Kings College, London, United Kingdom;

${ }^{* *}$ Institute of Cellular and Organismic Biology, Academia Sinica, Taipei, Taiwan;

${ }^{+\dagger}$ Enthought Inc, Austin, United States

Competing interests: The authors declare that no competing interests exist.

Funding: See page 12

Received: 05 September 2015 Accepted: 23 December 2015 Published: 24 December 2015

Reviewing editor: Moses $\mathrm{V}$ Chao, New York University School of Medicine, United States

(c) Copyright Zembrzycki et al. This article is distributed under the terms of the Creative Commons Attribution License, which permits unrestricted use and redistribution provided that the original author and source are credited.

\section{Genetic mechanisms control the linear scaling between related cortical primary and higher order sensory areas}

\author{
Andreas Zembrzycki ${ }^{1 *+}$, Adam M Stocker ${ }^{1+\ddagger}$, Axel Leingärtner $^{1 \S}$, \\ Setsuko Sahara ${ }^{11}$, Shen-Ju Chou ${ }^{1 * \star}$, Valery Kalatsky ${ }^{2++}$, Scott R May ${ }^{1}$, \\ Michael P Stryker², Dennis DM O'Leary ${ }^{1}$
}

\author{
${ }^{1}$ Molecular Neurobiology Laboratory, The Salk Institute for Biological Studies, La \\ Jolla, United States; ${ }^{2}$ Center for Integrative Neuroscience, Department of \\ Physiology, University of California, San Francsisco, San Francisco, United States
}

\title{
Introduction
}

The mouse neocortex is patterned into functionally distinct fields that include the primary sensory areas (visual, somatosensory and auditory), which receive modality-specific sensory inputs from thalamocortical axons (TCAs) originating from nuclei of the dorsal thalamus (O'Leary et al., 2013). In the cortex, the connections of TCAs establish precise topographic representations (or maps) of the sensory periphery (Krubitzer and Kaas, 2005; O'Leary et al., 2013). Primary areas are flanked by higher order sensory areas $(\mathrm{HO})$, which are interconnected with them and also contain topographic maps (Felleman and Van Essen, 1991). In mammals, this evolutionarily conserved general layout of the intra-areal neural circuits is responsible for the orderly progression of sensory information, sensory perception and the integration of higher cortical functions (Felleman and Van Essen, 1991; Geschwind and Rakic, 2013; Krubitzer and Kaas, 2005; Laramée and Boire, 2014; O'Leary et al., 2013). Disrupted layouts of cortical area layouts appear to be associated with neurodevelopmental disorders including autism (Courchesne et al., 2011; Voineagu et al., 2011). Studies of cortical arealization, the mechanisms that pattern the neocortex into areas, have focused almost exclusively on the primary areas and have led to the prevailing model that genetic mechanisms intrinsic to the neocortex control arealization during early cortical development (Greig et alı, 2013; Krubitzer and Kaas, 2005; O'Leary et al., 2013). For example, the graded expression of the homeodomain transcription factor Emx2 in neocortical progenitors determines the size and position of the primary visual area (V1) in mice (Bishop et al., 2000; Hamasaki et al., 2004). Although higher order areas outnumber primary areas by roughly 10-fold (Marshel et al., 2011; Wang and Burkhalter, 2007), 
eLife digest The neocortex is the most recently evolved part of the human brain. It is associated with higher thought processes, including language and the processing of information from our senses. Anatomically, the neocortex is organised into different regions called 'primary areas' and 'higher order areas', and perturbations to this organisation are associated with disorders such as autism.

There are many more higher order areas than primary areas in a mammalian brain. But, while primary areas are known to be specified by developmental genes in the embryo, little is known about how the development of higher order areas is controlled. Recent findings suggested that primary areas might themselves influence the emergence of higher order areas via a series of developmental events.

Now, Zembrzycki, Stocker et al. have investigated the developmental mechanisms that organise the mouse neocortex into its different regions. The experiments involved mouse mutants that produce either too much or too little of a protein called Emx2. This protein is known to determine the size and position of the primary visual area (commonly called V1) during embryonic development. In the mutant mice with too much $E m \times 2$, the primary visual area was about $150 \%$ larger than it should be, even though the neocortex was a normal size. Zembrzycki, Stocker et al. then went on to show that the higher order areas associated with the primary visual area also grew proportionally larger in these mutant mice. The opposite was true for mice that didn't produce Emx2 in their brains, and these mice had a much smaller primary visual area than normal mice.

Together, these findings reveal a previously unknown linear relationship between the size of the primary visual area and higher order visual areas that is controlled by the genes that pattern the neocortex during development. This and other new insights will inform future studies of the development and organization of the neocortex and our understanding of how it evolved. DOI: 10.7554/eLife.11416.002

mechanisms that specify them and define their proportions relative to primary areas have yet to be explored.

\section{Results}

To investigate the impact of altered primary area size on higher order areas, we have used the cortical visual area V1 as a model. Previous studies have shown that genetic manipulation of patterning genes, including Fgf17 and Emx2, results in altered V1 size (Cholfin and Rubenstein, 2007; Hamasaki et al., 2004). Here we have analyzed transgenic mice that overexpress Emx2 (ne-Emx2) and show area patterning defects including a V1 that is $\sim 150 \%$ of the normal size, while retaining overall normal neocortex size (Hamasaki et al., 2004 Leingårtner et al., 2007). By revealing the targeting patterns of TCAs projecting from thalamic sensory nuclei into the cortex (Fujimiya et al., 1986), the perimeters of primary sensory areas and the border between the neocortex and entorhinal cortex $(E C T)$ can be visualized by serotonin $(5 \mathrm{HT})$ staining using a single postnatal day $(\mathrm{P}) 7$ tangential section of the flattened cortical hemisphere (Figure 1A). The staining shows that, in addition to the previously reported enlarged V1 (Hamasaki et al., 2004,; Leingärtner et al., 2007), the cortical tissue that is nested between $\mathrm{V} 1$ and the surrounding primary areas (primary somatosensory cortex: S1, auditory areas: Aud) and the ECT laterally appears qualitatively larger in ne-Emx2 brains, when compared to wildtype (wt) sections (Figure 1A). We have defined this caudal cortical territory, which lies outside of $\mathrm{V} 1, \mathrm{~S} 1$, and the auditory areas and shows no or weak $5 \mathrm{HT}$ staining as a joint higher order cortical area complex and have termed it HO-5HT. The 5HTstaining revealed that this region contains the higher order visual areas surrounding V1 (Wang and Burkhalter, 2007), the retrosplenial cortex (RSC) medially, and the ventral posterior temporal cortex laterally. The accurate distribution of staining across cortical layers can only be estimated using tangential sections. However, using P7 sagittal section, we confirmed in layer 4 that the caudal 5HT-positive cortical area (corresponding to $\mathrm{V} 1$ ) and the anteriorly adjacent 5HT-negative area between V1 and S1 (corresponding to $\mathrm{HO}-5 \mathrm{HT}$ ) appears larger in ne-Emx2 brains than in wt ones (Figure 1B). Next, we labeled TCAs projecting to V1 by filling the dLG with crystals of the lipophilic neuronal tracer Dil. On 


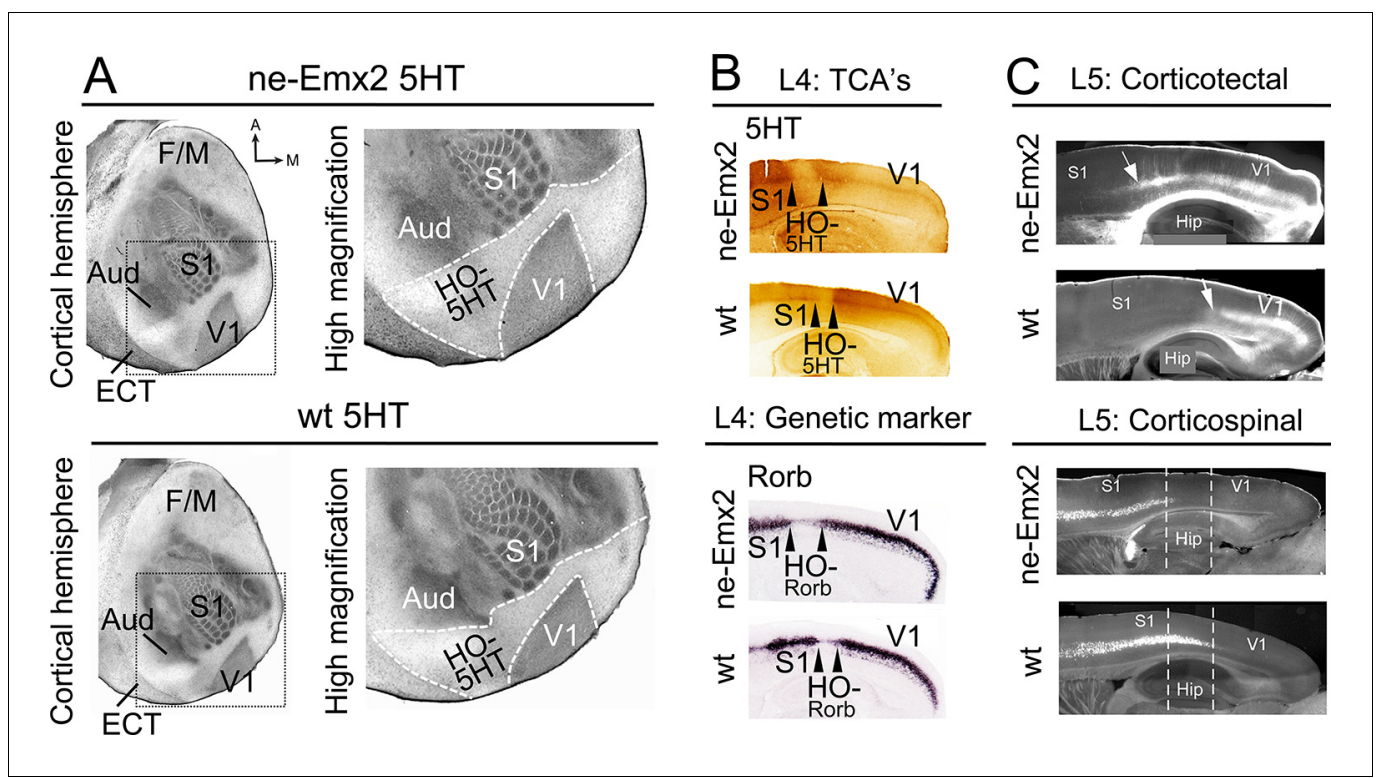

Figure 1. Increased V1 and higher order sensory area sizes in ne-Emx2 cortices (A) Serotonin (5HT) staining on postnatal day (P) 7 tangential sections of the flattened cortex reveals targeting patterns of TCAs revealing primary sensory area borders and the border of the neocortex to the ECT. 5HT staining is not detectable in the region containing the retosplenial cortex and the higher order sensory areas surrounding V1 (HO-5HT). In Emx2overexpressing brains (ne-Emx2), V1 and HO-5HT appear larger (compare dotted outlines in higher magnification images), compared to wt brains. (B) Targeting of TCAs in cortical layer 4 (L4) was revealed on P7 sagittal cortex sections by $5 \mathrm{HT}$ staining, whereas $\mathrm{L} 4$ genetic area borders were revealed by in situ hybridization for Rorb. In neEmx2 brains, the $\mathrm{V} 1$ border shifts anteriorly. Higher order areas surrounding $\mathrm{V} 1$ are characterized by low $5 \mathrm{HT} / \mathrm{Rorb}$ staining (between arrowheads, HO-5HT and HO-Rorb), which in ne-Emx2 brains appear overall larger (compare area between arrowheads). (C) In L5, an expansion (see arrows) of corticotectal projection neurons (retrogradely labeled by Dil injections into the superior colliculus) is apparent in ne-Emx2 brains, to the expense (see dotted lines) of L5 corticospinal projection neurons (retrogradely labeled by Dil injections into the pyramidal decussation). Main axes: A: anterior; M: medial; F/M: frontal/motor cortex; S1: primary somatosensory cortex; Aud: auditory areas; V1: primary visual cortex, ECT: entorhinal cortex. 5HT, serotonin; L5, cortical layer 5; TCAs, thalamocortical axons; wt, wildtype.

DOI: 10.7554/eLife. 11416.003

The following figure supplement is available for figure 1 :

Figure supplement 1. Anterior shifted cortical boundary formed by TCAs from the dLG in ne-Emx2 brains. DOI: 10.7554/eLife.11416.004

the P7 sagittal sections that were derived from five different medial to lateral levels, anterograde Dil labeling in the cortex revealed that TCAs from the dLG terminate in a smaller region in wt than in ne-Emx2 brains (Figure 1-figure supplement 1). Across genotypes, the Dil staining revealed a sharp border with adjacent cortical tissues that did not receive TCAs input from the dLG (Figure 1figure supplement 1). This finding is consistent with the $5 \mathrm{HT}$ staining and indicates a well-defined border between $\mathrm{V} 1$ neighboring higher order areas that is anteriorly shifted in ne-Emx2 brains.

Cortical areas can also be distinguished by area-specific gene expression patterns, which overlap with anatomical area borders and shift similarly when area patterning is disrupted (O'Leary et alo, 2013). For example, Rorb expression is strongly induced by thalamic input to primary areas (Jabaudon et al., 2012) like S1 and V1 but is low in areas that do not receive their major inputs from the principal thalamic sensory nuclei, such as cortical higher order areas surrounding V1 (Chou et al., 2013; Wang and Burkhalter, 2007). In situ hybridization (ISH) on sagittal sections adjacent to 5HTstained ones revealed sharp Rorb gene expression borders between areas in layer 4 . Notably in neEmx2 brains, the high-to-low Rorb expression border is located more anteriorly, and the area showing low Rorb expression and resembling HO-5HT (Chou et al., 2013) is larger than in wt sections (Figure 1B). This reveals that characteristic molecular markers that delineate the borders between 
V1 and surrounding higher order areas remain expressed at normal levels, but their sharp expression borders shift anteriorly in ne-Emx2.

Projection neurons in layer 5 , which extend axons into subcortical targets, are similarly determined by a molecular code (Greig et al., 2013). We therefore predicted that the areal shifts in neEmx2 brains would be accompanied by corresponding changes in layer 5 output projections. We labeled two distinct types of layer 5 subcerebral projection neurons by inserting Dil crystals either into the superior colliculus, which labels corticotectal projections from $\mathrm{V} 1$ and $\mathrm{HO}$, or else into the pyramidal decussation, which labels corticospinal projections from the frontal cortex and S1 (Greig et al., 2013; Zembrzycki et al., 2015). We found that layer 5 corticotectal projections extended more anteriorly in ne-Emx2 sagittal sections. Vice versa, the layer 5 corticospinal projections extended less posteriorly in ne-Emx2 brains. These staining patterns are consistent with an altered balance of projection neuron identity in layer 5 (Greig et al., 2013; Zembrzycki et al., 2015) and an overall expansion of visual areas, demonstrating that areal patterning changes in ne-Emx2 brains are not limited to the cortical layers that receive thalamic input. These findings complement previous reports describing Emx2 patterning functions (Bishop et al., 2000; Hamasaki et alı, 2004; Leingärtner et al., 2003; Leingärtner et al., 2007) and indicate for the first time that V1 and higher order area sizes are altered concomitantly in ne-Emx2 brains at the level of area-specific connectivity and gene expression in multiple cortical layers. Taken together, our results suggest that changes in primary area size are paralleled by similar changes in higher order area size.

It is commonly assumed that areal patterning changes also alter area-specific functional neuronal properties and topographic sensory maps, but this has never been demonstrated conclusively. Therefore, to compare functional neuronal properties of an enlarged visual cortex to a normal-sized one, we have used Fourier intrinsic signal optical imaging to construct topographic visual response maps to light bars that were moved across the visual field of the retina (up and down: elevation maps; left to right: azimuth maps) (Kalatsky and Stryker, 2003). Visual responses in V1 of wt and $n e-E m \times 2$ mice produced intrinsic signal maps that were indistinguishable in strength, and the axes of azimuth and elevation were organized in the same way in all tested brains (Figure 2A), revealing that functional topographic organization of the visual cortex was intact. However, the representations of elevation and azimuth were expanded in ne-Emx2 animals, and their retinotopic maps were overall larger (elevation: $138 \% \pm 8.7 \%$ of wt; azimuth: $143 \% \pm 8.2 \%$ of $w t)$. For example, the green region in the response maps representing $\sim 20$ to $\sim 30$ degrees of elevation/azimuth is clearly enlarged in ne-Emx2 brains, compared to wt brains (Figure 2A). To investigate the relationship between the location and size of the $\mathrm{V} 1$ functional response area and the histochemically delineated $\mathrm{V} 1$, as indicated by $5 \mathrm{HT}$ staining, multiple injections of Dil were placed lining the border of the V1 optical response map after the imaging procedure. On 5HT-stained, flattened tangential cortical sections, the Dil injection sites were found in all cases to be located near the border of the $5 \mathrm{HT}$ staining in $\mathrm{V} 1$, confirming the overall enlarged $\mathrm{V} 1$ perimeters in ne-Emx2 brains compared with the wt brains (Figure 2B). This shows that the 5HT-stained V1 area accurately corresponds to the intrinsic functional V1 map, suggesting that enlarged $\mathrm{HO}$ in ne-Emx2 brains have not acquired ectopic V1-like functional properties.

To further characterize the shifted border between visual areas in ne-Emx2 animals, we used additional neuronal tracing approaches. Stereotypically, V1 is connected with the dLG, whereas the HO areas are wired to the posterior thalamic nucleus (PO) (Leyva-Díaz and López-Bendito, 2013; López-Bendito and Molnár, 2003). To first label axonal connections between the cortex and the thalamus in wt brains, we administered dual tracer injections into locations that approximate to $\mathrm{HO}$ (DiD: green dye) and another injection around the approximated border area between $\mathrm{V} 1$ and $\mathrm{HO}$ (Dil: red dye). After diffusion of the tracers, we performed $5 \mathrm{HT}$ staining on flattened cortex sections to identify the areas in which the injections were administered and analyzed the patterns of retrograde dye labeling on coronal sections of the thalamus. In representative cases (Figure 2C) where 5HT staining confirmed that Dil was injected at the border between $\mathrm{V} 1$ and $\mathrm{HO}$ and DiD was injected into $\mathrm{HO}$, retrogradely labeled green DiD cells were apparent in the dLG and the PO. Conversely, red Dil cells were only labeled in the dLG. In ne-Emx2 brains, we administered similar dual tracer injections: Dil was targeted to $\mathrm{V} 1$ and a DiD injection was administered around the approximate border between $\mathrm{V} 1$ and $\mathrm{HO}$. Due to their enlarged V1, all ne-Emx2 injections were administered at more anterior coordinates than in wt brains (compare dashed lines in Figure 2C). In representative cases (Figure 2C) where 5HT staining confirmed that the Dil injection was 


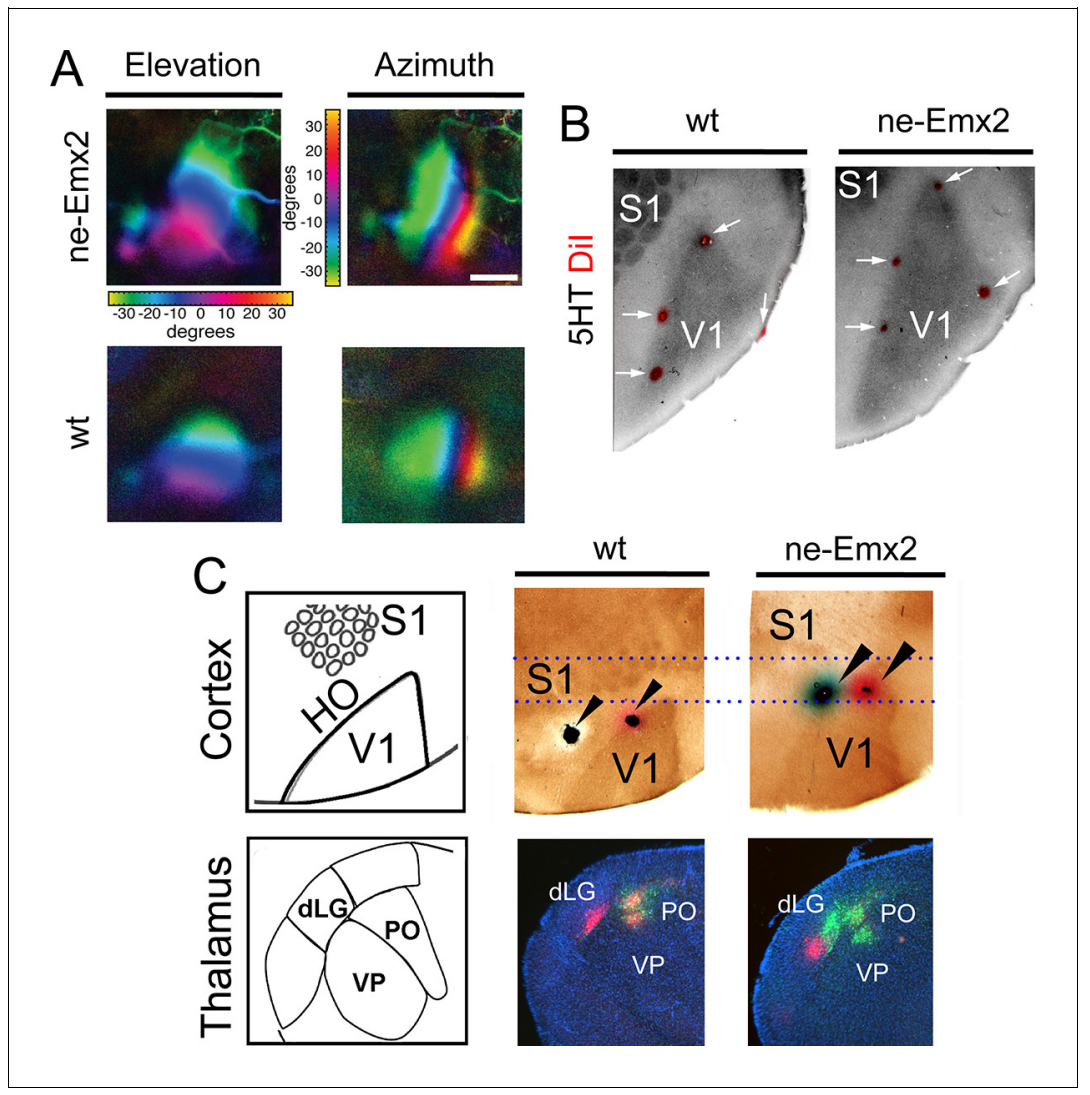

Figure 2. Enlarged functional V1 topographic maps in ne-Emx2 mice. (A) Fourier intrinsic signal optical imaging reveals topographic visual response maps to light bars that were moved across the visual field of the retina (up and down: elevation maps; left to right: azimuth maps). Visual responses in V1 produced intrinsic signal maps that were indistinguishable in strength and the color-coded axes of azimuth and elevation were organized in the same way in all tested brains (wt: $n=6, n e-E m \times 2: n=6$ ). The elevation/azimuth representations were expanded in neEmx2 animals, revealing that overall their $\mathrm{V} 1$ retinotopic maps were larger. (B) $5 \mathrm{HT}$ staining performed on flattened tangential cortex section reveals cortical area borders including $V 1$. On representative images $(n=6$ per genotype), the red dots lining the perimeter of the 5HT-stained V1 indicates Dil injection sites that were made after the recordings adjacent to the border of the derived V1 intrinsic response maps, determined by Fourier intrinsic optical imaging. (C) Schematics depict caudal cortical sensory areas and main sensory thalamus divisions. In wt brains ( $n=15)$, cortical dual tracer injections (red tracer (Dil) injected around V1/HO border; green tracer (DiD) into $\mathrm{HO}$; injection site location (arrowheads) was identified by $5 \mathrm{HT}$ staining) showed retrogradely labeled red cells in the dLG and the PO, whereas green labeled cells were only present in the PO. Dotted lines show that dual tracer injections in $n e-E m \times 2$ brains $(n=17)$ were administered at more anterior coordinates (red tracer into V1; green tracer around the $\mathrm{V} 1 / \mathrm{HO}$ border) compared with wt brains. In ne-Emx2 brains, retrogradely labeled red cells were apparent in the dLG, whereas green cells were present in the $\mathrm{dLG}$ and the PO, revealing normal thalamocortical connectivity patterns, but an anterior shifted $\mathrm{V} 1 / \mathrm{HO}$ border in ne-Emx2 brains. $5 \mathrm{HT}$, serotonin; dLG, dorsal lateral geniculate nucleus; PO, posterior thalamic nucleus; S1, primary somatosensory cortex; VP, ventroposterior nucleus; V1, primary visual cortex; wt, wildtype.

DOI: 10.7554/eLife.11416.005

administered into $\mathrm{V} 1$ and $\mathrm{DiD}$ was injected around the $\mathrm{V} 1 / \mathrm{HO}$ border, red cells were found in the dLG, whereas green-labeled cells were apparent in the PO and the dLG. The dual tracings in wt and ne-Emx2 brains are consistent with the predicted connectivity of cortical neurons around the injection sites (Leyva-Díaz and López-Bendito, 2013; López-Bendito and Molnár, 2003). Although located more anteriorly in ne-Emx2 brains, the subcortical connectivity patterns around the V1/HO borders were similar, demonstrating that these neurons show connectivity patterns that are consistent with their intrinsic areal identity and not their topographic location on the cortical sheet. Taken together, our results indicate that increased V1 size in ne-Emx2 brains is accompanied by a concomitant enlargement and anterior shift of $\mathrm{HO}$. 
To define the individual magnitudes of the $\mathrm{V} 1$ and $\mathrm{HO}$ size increases in ne-Emx2 brains, we next used gene expression domains as molecular markers delineating visual areas and quantified them (Figure 3). An accurate assessment of area sizes using flattened and/or sectioned cortical tissues could potentially be hampered by imperfect flattening of the tissues or by cutting artifacts. Therefore, we have used RNA in situ hybridization on intact whole brains (whole mount in situ hybridization: WMISH) at P7 for quantification purposes, which has the advantage that quantifications can be
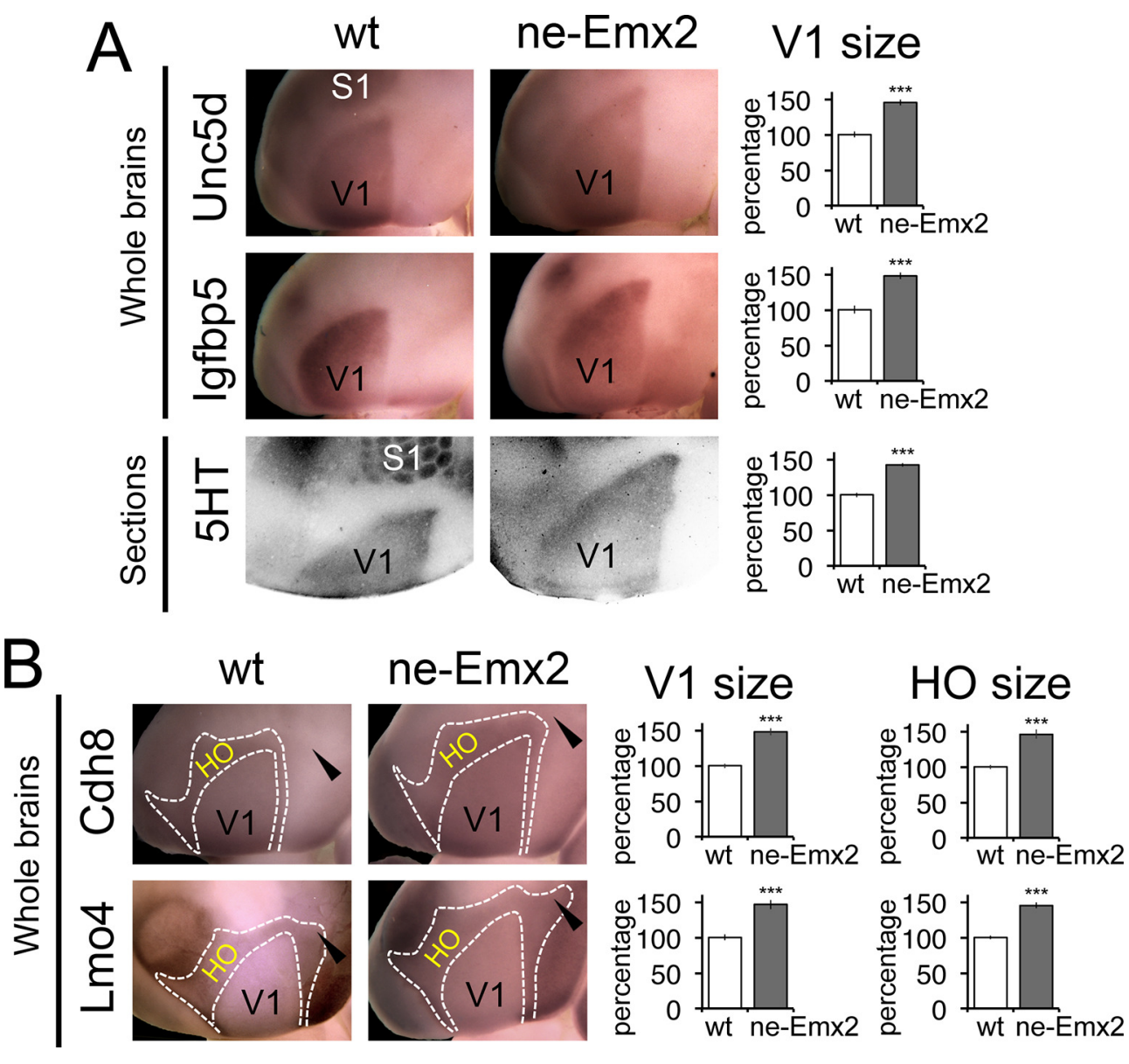

Figure 3. Proportionally increased $\mathrm{V} 1$ and $\mathrm{HO}$ sizes in ne-Emx2 cortices. (A) Schematic shows sensory area outlines in the caudal neocortex (12). WMISH with the molecular V1 marker genes Unc5d (wt: $n=5, n e-E m \times 2: n=6$ ) and Igfbp5 (wt: $n=11$, ne-Emx2: $n=6$ ) at P7 highlights increased V1 size in ne-Emx2 brains using whole un-sectioned brains. Quantification of V1 size using 5HT-stained P7 flattened cortical sections similarly reveals larger V1 sizes in ne-Emx2 brains $(n=11)$, compared to wt brains $(n=13)$. (B) WMISH for molecular markers that label both V1 and $\mathrm{HO}$ (dotted outlines: Cdh8, Lmo4: high expression in $\mathrm{HO}$, lower in $\mathrm{V} 1$; for each probe and genotype $\mathrm{n}=6$ ) reveal that $\mathrm{V} 1$ as well as $\mathrm{HO}$ sizes in ne-Emx2 are larger compared with wt brains. Cdh8 is not expressed around the anteromedial edge of V1 (arrowheads). Quantifications in Figures 3 and 4 show mean values as percent of wt, error bars indicate standard error of the mean; asterisks highlight statistical significance according to unpaired to t-test. 5HT, serotonin; S1, primary somatosensory cortex; WMISH, whole mount in situ hybridization; V1, primary visual cortex; wt, wildtype.

DOI: 10.7554/eLife.11416.006

The following figure supplements are available for figure 3:

Figure supplement 1. Lmo4 expression delineates primary and higher order cortical area boundaries in whole mount brains.

DOI: 10.7554/eLife.11416.007

Figure supplement 2. Lmo4 and Cdh8 expression marks primary and caudal extrastriate cortical regions.

DOI: 10.7554/eLife.11416.008 
made using single images without sectioning and artifacts that may arise from such tissue processing. We first used a set of two marker genes, Unc5d and Igfbp5, whose expression delineates V1 at P7 (Chou et alo, 2013). The gene expression domains on WMISH-stained brains were outlined and their sizes quantified as a measure of $\mathrm{V} 1$ area size. The mean value of wt brains was defined as $100 \%$ and the area size percentages of ne-Emx2 brains displayed accordingly as 'percent of $\mathrm{wt}^{\prime}$ (Figure 3A). V1 gene expression domains of both markers were larger in ne-Emx2 brains (Unc5d-V1: $148 \% \pm 6.1 \%, \mathrm{p}=0.0003$; Igfbp5-V1: $148 \pm 4.5 \%, \mathrm{p}<0.0001$ ). The magnitude of the increased in $\mathrm{V} 1$ size labeled genetically in ne-Emx2 brains is comparable to $\mathrm{V} 1$ area measurements derived from 5HT-stained P7 flattened cortical sections (Figure 3A: 5HT-V1: 142.1\% $\pm 3.1 \%, \mathrm{p}<0.0001$ ), indicating that molecular markers on whole brains reliably delineate $\mathrm{V} 1$ and can therefore be used to quantify and compare area sizes between samples and mouse lines.

We next have used additional markers to quantify higher order area sizes (Figure 3B). Previous studies have parsed higher order visual areas using neuroanatomical tracers (Wang and Burkhalter, 2007) (see also schematic in Figure 3) and have revealed genes that are expressed at different levels in V1 and higher order visual areas (Chou et alo, 2013). For example, Cdh8 and Lmo4 expression is higher in the area surrounding V1, where higher order visual areas are located (Chou et alo, 2013; Marshel et al., 2011; Wang and Burkhalter, 2007). The domains of high Cdh8 and Lmo4 expression appear to label higher order visual areas uniformly (Chou et al., 2013), without revealing subdivisions between them (compare schematic in Figure 3 showing approximate location and outline of higher order visual areas as identified by Wang and Burkhalter, 2007 to Cdh8 and Lmo4 gene expression domains around V1). On P7 WMISH-stained wt brains, we quantified the V1 and HO sizes in the medial cortex on the basis of low and high gene expression domains (see dotted lines in Figure 3, Figure 3-figure supplement 2). Anatomically, these gene expression domains surrounding $\mathrm{V} 1$, which show much stronger staining compared with $\mathrm{V} 1$, extend anteriorly up to the S1 border, laterally to the border of the auditory areas and the ECT and medially up to the border to the RSC, respectively (Figure 3-figure supplements 1,2). Hence, compared with the above-mentioned HO complex that was identified using $5 \mathrm{HT}$ staining (Figure 1), the higher order area complex labeled by $\mathrm{Cdh} 8$ and $\mathrm{Lmo} 4$ relates to a smaller cortical region that more closely relates to higher order visual areas, but excludes the RSC. The size (Cdh8-V1: $145.3 \pm 7.4 \%, \mathrm{p}<0.0001 ;$ Lmo4-V1: $146.6 \% \pm 6.7 \%$, $\mathrm{p}=0.0005$ ) and shape of the gene expression domains in V1 were similar in Cdh8- and Lmo4-stained brains. Similarly, the gene expression domains nested around V1 largely overlapped between the two probes. The only apparent difference between them is around the anteromedial edge of the higher order visual areas (Wang and Burkhalter, 2007), where Cdh8 is expressed at much lower levels compared to more lateral regions around V1 across genotypes (arrowheads in Figure 3B). The wt values of the measurements were again defined as $100 \%$. The overall shapes of the two HO marker gene domains were similar and the sizes larger in ne-Emx2 brains compared with wt brains (Cdh8- HO: $145.7 \pm 6.4 \%, p=0.0015$; Lmo4- HO: $144.9 \pm 3.8 \%, p=0.00157$ ). The analysis of different area-specific sets of marker genes, either showing unique expression in $\mathrm{V} 1$, or discernable expression levels between visual areas, revealed an increase in visually-related $\mathrm{HO}$ in ne-Emx2 brains that was proportionate to the V1 size increase. The extrastriate areas that we have identified on the basis of $5 \mathrm{HT}$ staining (Figure 1) included the RSC, which is not a higher order visual area (Garrett et al., 2014; Marshel et al., 2011; Vann et al., 2009; Wang and Burkhalter, 2007) raising the possibility that only related primary and higher order areas (e.g. vision) could scale proportionately. To test this possibility, we have used WMISH of Lypd1 on P7 wt and ne-Emx2 brains as a specific marker labeling the caudomedial cortex, where the RSC is located (Figure 3-figure supplement 1). We found that the specific Lypd1 gene expression domain in the caudomedial cortex is significantly enlarged in ne-Emx2 brains $(114.3 \pm 5.2 \%, p=0.0225, n=4)$, compared with wt brains. This size increase is not proportionate to the size increases of $\mathrm{V} 1$ and the higher order visual area complex labeled by Cdh8 and Lmo4 in ne-Emx2 brains (Figure 3) suggesting that increased V1 size is specifically accompanied by a proportionate size increase of related higher order visual areas.

To test if related $\mathrm{HO}$ size matches $\mathrm{V} 1$ size only when it is larger than normal, or if primary area size bi-directionally is accompanied by according scaling of related higher order areas, we next analyzed $\mathrm{HO}$ sizes excluding the RSC in brains with a smaller than normal V1 (Figure 4). Constitutive Emx2 mutant mice have an overall smaller brain and visual cortex, but homozygous mutants die perinatally (Bishop et al., 2000), preventing the analysis of cortical areas, which arise at later stages. To overcome this limitation, we generated a novel mouse line with floxed Emx2 alleles (Figure 4- 


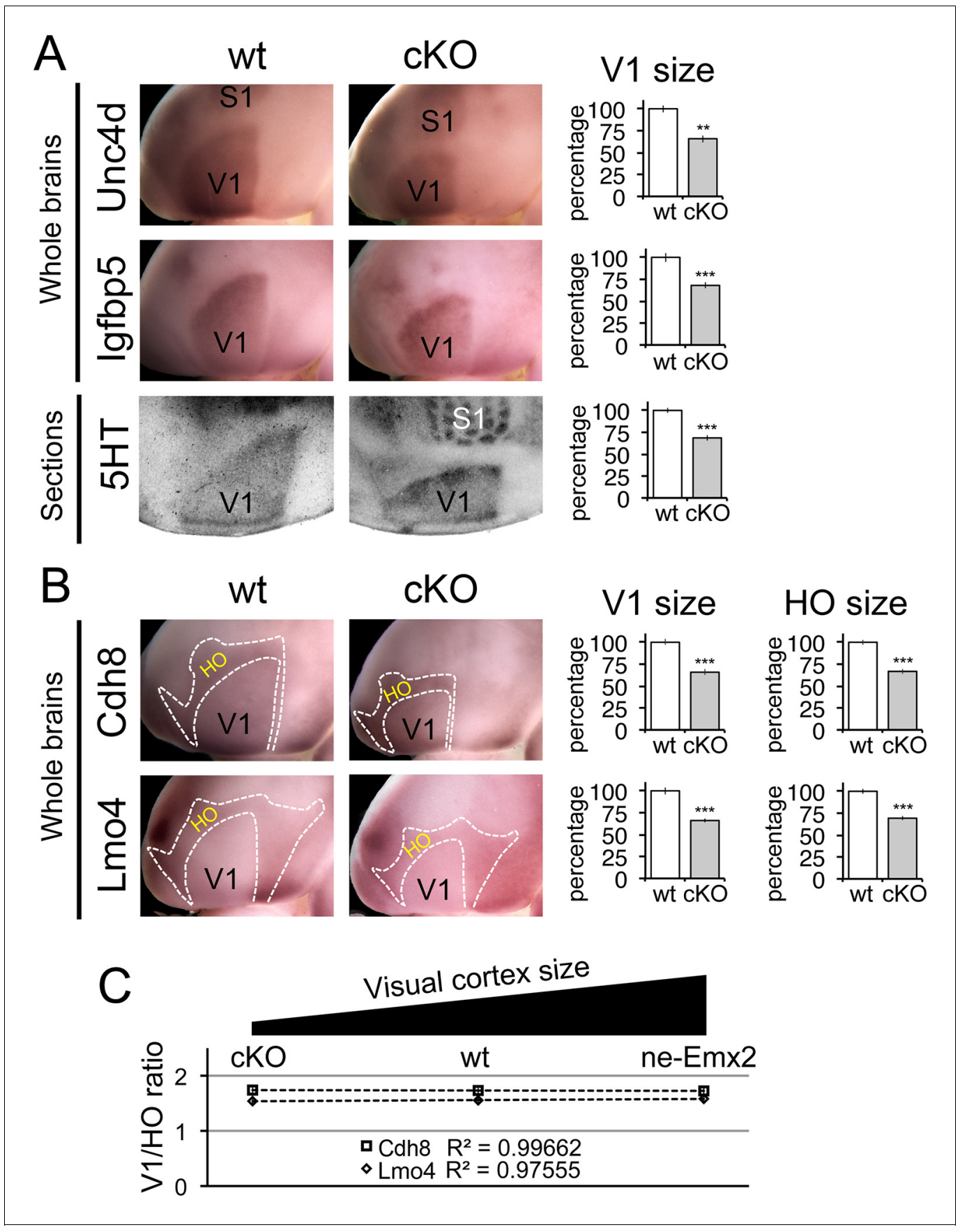

Figure 4. Proportionally decreased $V 1$ and $H O$ sizes in $c K O$ cortices. WMISH for V1 (A: Unc5d; wt: $n=5$, ne-Emx2: $n=6$, Igfbp5; wt: $n=11$, ne-Emx2: $n$ $=8$ ) or $\mathrm{V} 1$ and $\mathrm{HO}$ marker genes (B: Cdh8; wt: $n=6$, ne-Emx2: $n=6$; Lmo4; wt: $n=5$, ne-Emx2: $n=5)$ at P7 conversely reveals decreased sizes ( 70\% of wt size) of $\mathrm{V} 1$ and $\mathrm{HO}$ in brains that were derived from Emx1-IRES-Cre-mediated cortex-specific conditional Emx2 mutant brains (cKO), compared with wt brains. Quantification of V1 size using 5HT staining on P7 flattened cortical sections (A: wt: $n=15, c K O: n=10$ ) reveals similar reductions of V1 size in $c K O$ brains. (C) The ratio between quantified $\mathrm{V} 1$ and $\mathrm{HO}$ sizes derived from WMISH-stained brains with decreased (cKO), normal (wt), and increased (ne-Emx2) V1 sizes demonstrates linear scaling of $\mathrm{HO}$ size in response to bi-directional changes of $\mathrm{V} 1$ size. S1, primary somatosensory cortex; V1, primary visual cortex; WMISH, whole mount in situ hybridization; wt, wildtype.

DOI: 10.7554/eLife.11416.009

The following figure supplements are available for figure 4:

Figure supplement 1. Generation of Emx2-floxed mice and confirmation of cortex specific Emx2 deletion.

DOI: 10.7554/eLife.11416.010

Figure supplement 2. Normal cortical neuroanatomy in $c K O$ brains.

Figure 4 continued on next page 
Figure 4 continued

DOI: 10.7554/eLife.11416.011

Figure supplement 3. Area patterning changes following cortex-specific deletion of Emx2.

DOI: 10.7554/eLife.11416.012

Figure supplement 4. Posteriorly shifted V1 border in cKO cortices.

DOI: 10.7554/eLife.11416.013

figure supplement 1), allowing conditional inactivation of Emx2. We crossed Emx2 floxed mice with Emx1-IRES-Cre expressing mice (Gorski et al., 2002) to generate conditional, cortex-specific Emx2 mutant mice. These cKO mice are viable, fertile and have an anatomically normal neocortex (Figure 4-figure supplement 2). Confirming the prediction that reduced Emx2 expression levels in cortical progenitors would lead to smaller visual areas, cKO brains show areal patterning changes (Figure 4-figure supplement 3) that are similar to those previously reported in heterozygous Emx2 mutant brains (e.g. larger frontal cortex) (Hamasaki et al., 2004), but are opposite to those apparent in ne-Emx2 brains (e.g. smaller frontal cortex) (Hamasaki et al., 2004; Leingärtner et al., 2007). As in ne-Emx2 brains, $\mathrm{V} 1$ in $c K O$ brains was characterized using $5 \mathrm{HT}$ staining and Dil injections into the dLG (Figure 4-figure supplement 4), revealing that V1 in $c K O$ is greatly reduced in size.

To complement the quantification of $\mathrm{V} 1$ and its related $\mathrm{HO}$ sizes in ne-Emx2 brains, we next have used $\mathrm{cKO}$ brains to perform WMISH with both sets of marker genes noted above (Figure 3) and measured their sizes (Figure 4). Measurements of the molecular V1 marker domains (Unc5d-V1: 67.1 $\pm 2.6 \%, \mathrm{p}=0.00029$; I ffbp5-V1: $68 \pm 3.3 \%, \mathrm{p}=0.00017$ ), as well as the $\mathrm{V} 1$ expression domains of Cdh8 (67.7 $\pm 4.5 \%, p<0.0001)$ and Lmo4 (68.6 $\pm 0.8 \%, p<0.0001)$ revealed that the molecularly defined V1 in $c K O$ was smaller than in wt brains. These reductions matched the reduced V1 in $5 \mathrm{HT}$ stained flattened cortex sections (Figure 4A: 5HT-V1: $68.5 \pm 3 \%, p<0.0001$ ). Subsequent quantification of the gene expression domains of Cdh8 and Lmo4 surrounding V1 (Figure 4B: Cdh8- HO: $66.8 \pm 2.9 \%, \mathrm{p}<0.0001$; Lmo4- $\mathrm{HO}: 69.3 \pm 2.4 \%, \mathrm{p}<0.0001)$ revealed that the cortical region that contains visually-related $\mathrm{HO}$ was also reduced in $c K O$ brains to a degree proportional to the reduction in $\mathrm{V} 1$ size. These data demonstrate that when $\mathrm{V} 1$ size is reduced, related $\mathrm{HO}$ size is reduced to a similar extent.

In order to reveal a correlation between primary and related higher order area size between brains with larger and smaller than normal visual areas, we calculated the ratios between the genetically defined $\mathrm{V} 1$ and related $\mathrm{HO}$ sizes (Figure $4 \mathrm{C}$ ). Ranging over an $\sim 80 \%$ variation of the normal V1 size, Figure $4 \mathrm{C}$ reveals that related $\mathrm{HO}$ size is bi-directionally altered in a linear fashion (Cdh8 regression: $y=-0.0071 x+1.7463 ;$ Lmo4 regression: $y=0.0208+1.7463)$. Taken together, our results are consistent with a proportional scaling relationship between the size of primary and related higher order visual areas: The size of V1 is determined by the activity of transcription factors including Emx2 during development, and this mechanism likewise controls the linear matching of the proportions of higher order visual areas in the mouse neocortex.

\section{Discussion}

The present findings address the mechanisms that specify and regulate the size of higher order sensory areas, an issue that has been largely neglected. They reveal a novel, prominent role for intrinsic genetic information in this process. Genetically altering the size of V1 over a range of $\sim 80 \%$ of its normal size using a Emx2 gain-of function mouse line and a novel conditional Emx2 loss-of function mouse line showed that the specification of both primary and related higher order cortical areas during development was linearly scaled by driving the unique properties that characterize both, V1 and higher order visual areas.

Regardless of whether V1 was larger or smaller than in wt mice, related $\mathrm{HO}$ exhibited normal cytoarchitecture, genetic profiles, functional properties, and characteristic patterns of connectivity that resulted in an overall uniformly altered 'visual cortical field' in the occipital cortex that remained accurately and proportionally subdivided into V1 and higher order visual areas. This demonstrates that Emx2 (and perhaps additional intrinsic area patterning regulators) specify a 'sensory cortical field' that includes primary and higher order areas and a defined border between them. This model 
of cortical area patterning is not consistent with the possibility that the core properties of primary and higher order areas are specified sequentially or through parallel genetic mechanisms.

Our results further reveal that higher order areas do not have a fixed size. Rather their relative size is flexible. By using mouse models with bi-directional changes of V1 size as a model, our study revealed that higher order areas scale linearly together with their related primary sensory areas, This observation is important for at least two reasons: (i) it re-emphasizes a sequential model of primary sensory area formation that likewise influences the properties of related higher order areas. In this model, cortical intrinsic mechanisms specify all generic primary and higher order visual cortex properties during early development. Much later during postnatal development, geniculocortical input is needed to terminally differentiate the genetic distinctions between V1 and $\mathrm{HO}$ (Chou et alo, 2013; Vue et alo, 2013). (ii) It contradicts the hypothesis that cortical structure/function evolution mainly is driven by a disproportionate increase in the size of related higher order areas relative to primary areas. To the contrary, our results show that primary and related higher order areas remain proportionate when primary area size is altered through genetic mechanisms, suggesting that an increase in the complexity of connections and micro-circuits among higher order cortical processing centers likely accounts for gains in cortical functions that are characteristic for gyrencephalic mammals with larger cortical surface areas, compared to simpler lissencephalic mammals. In summary, the newly discovered linear scaling relationship between primary and related higher order areas has major implications for the basic understanding of the development and organization of the neocortical bauplan and its evolution and variability in normal and affected conditions.

\section{Materials and methods}

\section{Mouse lines and conditional Emx2 gene targeting}

All experiments were approved and conducted following the guidelines of the Institutional Animal Care and Use Committee at the Salk Institute and were in full compliance with the guidelines of the National Institutes of Health for the care and use of laboratory animals. When mice were mated, the morning of the identified vaginal plug was designated as E0.5. The morning on which pups were born was designated P 0.5. Transgenic mice overexpressing Emx2 under the Nestin promoter (neEmx2) were previously described (Hamasaki et al., 2004). For generating Emx2 floxed mice $(E m \times 2 f / / f l)$, gene targeting was carried out using homologous recombination in embryonic stem cells. A targeting construct was designed in which the $5^{\prime} \operatorname{lox} P$ site was upstream of the Emx2 transcriptional start site and the $3^{\prime}$ loxP site downstream of Exon 2, followed by a FRT-site-flanked PGK-Neo cassette, Figure 4-figure supplement 1). Targeted embryonic stem cell clones were screened by Southern blot with probes A, B, and C and by PCR to identify Emx2floxed-neo/+ clones (Figure 4figure supplement 1). Positive clones were injected into C57BL/6J blastocysts at the Salk Transgenic Core Facility and chimeras were mated to C57BL/6J females to obtain germline transmission. Heterozygous mice were mated with mice expressing FLPe (Rodríguez et al,, 2000) to remove the neo

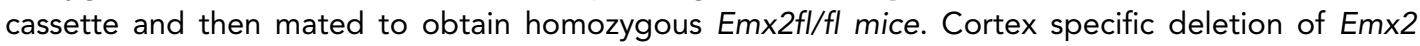
(cKO) was obtained by crossing Emx2fl/fl mice with Emx1-IRES-Cre mice (Gorski et alo, 2002). Specificity of Emx1-IRES-Cre-mediated deletion of Emx2 floxed alleles was analyzed by WMISH (described below) staining using a full-length Emx2 antisense RNA probe on E11 embryos. Genotyping was performed using primers for $E m \times 2$ floxed alleles ( $E m \times 2$ forward: GAC-TCC-TTTCCC-AAA-TAA-CCC-C, Emx2 reverse: GTA-AGC-GGG-CGG-GGA-CTG-GTT-C) and for the Cre recombinase (cre forward: GCT-AAA-CAT-GCT-TCA-TCG-TCG-G, cre reverse: GAT-CTC-CGG-TATTGA-AAC-TCC-AGC), and the ne-Emx2 transgene (nestin forward: TCA-ACC-CCT-AAA-AGC-TCC, Emx2 reverse: GGA-CGG-AGA-GAA-GGC-GGT).

\section{In situ hybridization, immunostainings, and tangential cortical sections}

Tissues were dissected, washed in phosphate-buffered saline (PBS), fixed overnight in $4 \%$ phosphate-buffered paraformaldehyde (PFA), washed in PBS, and cryopreserved in $30 \%$ sucrose in PBS. Postnatal brains were perfused with PFA, postfixed overnight in PFA, washed with PBS, and cryopreserved in 30\% sucrose in PBS. Tissues were embedded in Tissue-Tek OCT (Sakura Finetek, Japan) and sectioned on a cryostat (Leica, Germany). Antisense RNA probes were labeled using a DIG-RNA labeling kit (Roche, Switzerland). ISH on 18- $\mu \mathrm{m}$ cryostat sections and WMISH using P7 brains were 
carried out as previously described (Chou et al., 2013; Hamasaki et al., 2004; Zembrzycki et al., 2007; Zembrzycki et al., 2015). For tangential cortical sections, cortical hemispheres were dissected, flattened, postfixed between slide glasses, and then cryoprotected. Tangential sections were cut into $40-\mu \mathrm{m}$ slices from flattened cortical hemispheres with a sliding microtome and then they were immunostained for Serotonin (5HT, ImmunoStar, Hudson, WI). Immunostaining was developed using the diaminobenzidine colorimetric reaction and the Vectastain kit (Vector, Burlingame, CA). For Nissl staining, sections were stained with $0.5 \%$ cresyl violet and then dehydrated with graded alcohols.

\section{Axon tracings}

Lipophilic tracers Dil and DiD (all from Molecular Probes, Eugene, OR) were used to label corticothalamic-, thalamocortical-, corticotectal-, and corticospinal projections. For each experiment 4-6 brains with comparable tracer injection sites were cut and used for further data analysis, representative example images are shown in the figures. Analysis of thalamocortical axons by thalamic Dil injections (Figure 1B): P7 brains were fixed in 4\% PFA, hemisected, and a coronal cut between the diencephalon and mesencephalon was made in order to expose thalamic nuclei at the section surface and Dil crystals were implanted to cover the dorsal lateral geniculate nucleus (dLG). After incubation for 1 to 2 months at $30^{\circ} \mathrm{C}$ to $60^{\circ} \mathrm{C}$, preparations were sectioned sagittally on a vibratome (Leica). Sections were counterstained with DAPI (Vector) and analyzed under a fluorescence microscope to determine the tangential distribution of labeled thalamocortical axons in the neocortex.

Analysis of layer 5 subcerebral projection neurons (Figure 1C): Corticospinal neurons in cortical layer 5 were retrogradely labeled by inserting Dil crystals into the pyramidal decussation in 4\% PFA fixed brains. Layer 5 corticotectal neurons were labeled in 4\% PFA fixed brains by implantation of small Dil crystals into the upper layers of superior colliculus. Brains were incubated at $37^{\circ} \mathrm{C}$ for $2-3$ months before $100 \mu \mathrm{m}$ sagittal vibratome sections were cut and analyzed under fluorescent light. Analysis of area-specific thalamocortical and corticothalamic connectivity of caudal cortex (Figure 2): P7 pups were anesthetized by hypothermia and a small area of skull was removed to expose the cortical surface. Dil crystals and a small piece of DiD were implanted into cortical locations around V1 and the $\mathrm{V} 1 / \mathrm{HO}$ border. After 1 day of survival, brains were removed after $4 \%$ PFA perfusion and their cortices and thalami dissected. Cortices were then flattened and stained for 5-HT to reveal primary sensory areas relative to the dye injection sites. The thalami were preserved sectioned coronally, stained with DAPI and Dil/DiD labeled cells analyzed under a fluorescence microscope.

\section{Statistical analysis, area measurements, and intrinsic signal optical imaging}

Data collection and analyses were performed blind to genotype and the conditions of the experiments, data were collected and processed randomly, and no data points were excluded. No statistical methods were used to predetermine sample sizes, but our sample sizes were similar to those reported in previous publications (for example, (Chou et al., 2013; Zembrzycki et al., 2013). Data met the assumptions of the statistical tests used, and the data distribution was assumed to be normal but was not formally tested. Statistics were calculated with Microsoft Excel. Quantifications show mean values of the tested groups and are displayed as a percentage of the wt group. Quantified sample sizes (number of brains: $n$ ) are indicated in the figure legends. The examples shown in each figure are representative and were reproducible for each set of experiments. Individual experiments were successfully repeated at least three times using different litters.

Area size measurements on $5 \mathrm{HT}$ stained sections and statistical analysis was performed as previously described (Leingärtner et al., 2007; Zembrzycki et al., 2015; 2013). To quantify molecular V1 and HO sizes, gene expression domains were quantified on single images of WMISH-stained brains (examples of measured area outlines are shown as dashed lines in Figures 3,4 ) using ImageJ (Rasband 1997-2013). Derived wt mean values were defined as $100 \%$ and values of the other mouse lines calculated accordingly. Statistical significance was determined using unpaired two-tailed t test, $\mathrm{p}$ values $<0.05$ (indicated as *) were considered as statistically significant. Variance is indicated in the main text sections reflecting standard error of the mean. Intrinsic signal optical imaging was performed as previously described (Kalatsky and Stryker, 2003). To determine the spatial relationship between V1 functional maps and V1, defined histochemically by 5-HT staining, animals were imaged 
to determine the V1 map, and after completion, small Dil injections were made outside of the functional map perimeters. Animals were then perfused with $4 \%$ PFA, cortices dissected, flattened, sectioned tangentially, and stained for $5-\mathrm{HT}$.

\section{Acknowledgements}

We thank Kevin Jones for providing Emx1-IRES-CRE mice. This work was supported by the Vincent $\mathrm{J}$ Coates Chair of Molecular Neurobiology at the Salk Institute for Biological Sciences (DDMO).

\section{Additional information}

Funding

\begin{tabular}{lll} 
Funder & Grant reference number & Author \\
\hline National Institutes of Health & NS31558 & Dennis DM O'Leary \\
\hline National Institutes of Health & MH086147 & Dennis DM O'Leary \\
\hline National Institutes of Health & EY02874 & Michael P Stryker \\
\hline
\end{tabular}

The funders had no role in study design, data collection and interpretation, or the decision to submit the work for publication.

\section{Author contributions}

AZ, Conception and design, Acquisition of data, Analysis and interpretation of data, Drafting or revising the article; AMS, Acquisition of data, Analysis and interpretation of data, Drafting or revising the article; AL, SS, SJC, VK, SRM, Acquisition of data, Analysis and interpretation of data; MPS, Analysis and interpretation of data, Drafting or revising the article; DDMO'L, Conception and design, Analysis and interpretation of data, Drafting or revising the article

\section{Author ORCIDs}

Andreas Zembrzycki, (iD http://orcid.org/0000-0001-8468-4195

Ethics

Animal experimentation: All experiments were approved under Protocol \#09-012 and conducted following the guidelines of the Institutional Animal Care and Use Committee at the Salk Institute and were in full compliance with the guidelines of the National Institutes of Health for the care and use of laboratory animals.

\section{References}

Bishop KM, Goudreau G, O'Leary DD. 2000. Regulation of area identity in the mammalian neocortex by Emx2 and Pax6. Science 288:344-349. doi: 10.1126/science.288.5464.344

Cholfin JA, Rubenstein JL. 2007. Patterning of frontal cortex subdivisions by Fgf17. Proceedings of the National Academy of Sciences of the United States of America 104:7652-7657. doi: 10.1073/pnas.0702225104

Chou S-J, Babot Z, Leingartner A, Studer M, Nakagawa Y, O'Leary DDM. 2013. Geniculocortical input drives genetic distinctions between primary and higher-order visual areas. Science 340:1239-1242. doi: 10.1126/ science. 1232806

Courchesne E, Mouton PR, Calhoun ME, Semendeferi K, Ahrens-Barbeau C, Hallet MJ, Barnes CC, Pierce K. 2011. Neuron number and size in prefrontal cortex of children with autism. JAMA 306:2001-2010. doi: 10. 1001/jama.2011.1638

Felleman DJ, Van Essen DC. 1991. Distributed hierarchical processing in the primate cerebral cortex. Cerebral Cortex 1:1-47. doi: 10.1093/cercor/1.1.1

Fujimiya M, Kimura H, Maeda T. 1986. Postnatal development of serotonin nerve fibers in the somatosensory cortex of mice studied by immunohistochemistry. The Journal of Comparative Neurology 246:191-201. doi: 10. 1002/cne.902460205

Garrett ME, Nauhaus I, Marshel JH, Callaway EM. 2014. Topography and areal organization of mouse visual cortex. Journal of Neuroscience 34:12587-12600. doi: 10.1523/JNEUROSCI.1124-14.2014

Geschwind DH, Rakic P. 2013. Cortical evolution: judge the brain by its cover. Neuron 80:633-647. doi: 10.1016/ j.neuron.2013.10.045 
Gorski JA, Talley T, Qiu M, Puelles L, Rubenstein JL, Jones KR. 2002. Cortical excitatory neurons and glia, but not GABAergic neurons, are produced in the Emx1-expressing lineage. The Journal of Neuroscience: The Official Journal of the Society for Neuroscience 22:6309-6314.

Greig LC, Woodworth MB, Galazo MJ, Padmanabhan H, Macklis JD. 2013. Molecular logic of neocortical projection neuron specification, development and diversity. Nature Reviews. Neuroscience 14:755-769. doi: 10.1038/nrn3586

Hamasaki T, Leingärtner A, Ringstedt T, O'Leary DD. 2004. EMX2 regulates sizes and positioning of the primary sensory and motor areas in neocortex by direct specification of cortical progenitors. Neuron 43:359-372. doi: 10.1016/j.neuron.2004.07.016

Jabaudon D, J. Shnider S, J. Tischfield D, J. Galazo M, Macklis JD. 2012. ROR induces barrel-like neuronal clusters in the developing neocortex. Cerebral Cortex 22:996-1006. doi: 10.1093/cercor/bhr182

Kalatsky VA, Stryker MP. 2003. New paradigm for optical imaging: temporally encoded maps of intrinsic signal. Neuron 38:529-545.

Krubitzer L, Kaas J. 2005. The evolution of the neocortex in mammals: how is phenotypic diversity generated? Current Opinion in Neurobiology 15:444-453. doi: 10.1016/j.conb.2005.07.003

Laramée ME, Boire D. 2014. Visual cortical areas of the mouse: comparison of parcellation and network structure with primates. Frontiers in Neural Circuits 8:149. doi: 10.3389/fncir.2014.00149

Leingärtner A, Richards LJ, Dyck RH, Akazawa C, O'Leary DD. 2003. Cloning and cortical expression of rat Emx2 and adenovirus-mediated overexpression to assess its regulation of area-specific targeting of thalamocortical axons. Cerebral Cortex 13:648-660. doi: 10.1093/cercor/13.6.648

Leingärtner A, Thuret S, Kroll TT, Chou SJ, Leasure JL, Gage FH, O'Leary DD. 2007. Cortical area size dictates performance at modality-specific behaviors. Proceedings of the National Academy of Sciences of the United States of America 104:4153-4158. doi: 10.1073/pnas.0611723104

Leyva-Díaz E, López-Bendito G. 2013. In and out from the cortex: development of major forebrain connections. Neuroscience 254:26-44. doi: 10.1016/j.neuroscience.2013.08.070

López-Bendito G, Molnár Z. 2003. Thalamocortical development: how are we going to get there? Nature Reviews Neuroscience 4:276-289. doi: 10.1038/nrn1075

Marshel JH, Garrett ME, Nauhaus I, Callaway EM. 2011. Functional specialization of seven mouse visual cortical areas. Neuron 72:1040-1054. doi: 10.1016/j.neuron.2011.12.004

O'Leary DDM, Stocker AM, Zembrzycki A. 2013. Area Patterning of the Mammalian Cortex. In: O'Leary DDM, Stocker AM, Zembrzycki A, John Rubenstein, Pasko Rakic Patterning and Cell Type Specification in the Developing Cns and Pns. Oxford: Elsevier; 61-85. doi: 10.1016/B978-0-12-397265-1.00021-6

Rasband WS. 1997-2013. ImageJ, U. S. National Institutes of Health, Bethesda, Maryland, USA. U. S. National Institutes of Health. http://imagej.nih.gov/ij/

Rodríguez Cl, Buchholz F, Galloway J, Sequerra R, Kasper J, Ayala R, Stewart AF, Dymecki SM. 2000. Highefficiency deleter mice show that FLPe is an alternative to cre-loxP. Nature Genetics 25:139-140. doi: 10.1038/ 75973

Vann SD, Aggleton JP, Maguire EA. 2009. What does the retrosplenial cortex do? Nature Reviews Neuroscience 10:792-802. doi: 10.1038/nrn2733

Voineagu I, Wang X, Johnston P, Lowe JK, Tian Y, Horvath S, Mill J, Cantor RM, Blencowe BJ, Geschwind DH. 2011. Transcriptomic analysis of autistic brain reveals convergent molecular pathology. Nature 474:380-384. doi: 10.1038/nature10110

Vue TY, Lee M, Tan YE, Werkhoven Z, Wang L, Nakagawa Y. 2013. Thalamic control of neocortical area formation in mice. Journal of Neuroscience 33:8442-8453. doi: 10.1523/JNEUROSCI.5786-12.2013

Wang Q, Burkhalter A. 2007. Area map of mouse visual cortex. The Journal of Comparative Neurology 502:339357. doi: 10.1002/cne.21286

Zembrzycki A, Griesel G, Stoykova A, Mansouri A. 2007. Genetic interplay between the transcription factors Sp8 and $E m \times 2$ in the patterning of the forebrain. Neural Development 2:8. doi: 10.1186/1749-8104-2-8

Zembrzycki A, Chou SJ, Ashery-Padan R, Stoykova A, O'Leary DD. 2013. Sensory cortex limits cortical maps and drives top-down plasticity in thalamocortical circuits. Nature Neuroscience 16:1060-1067. doi: 10.1038/nn.3454

Zembrzycki A, Perez-Garcia CG, Wang CF, Chou SJ, O'Leary DD. 2015. Postmitotic regulation of sensory area patterning in the mammalian neocortex by Lhx2. Proceedings of the National Academy of Sciences of the United States of America 112:6736-6741. doi: 10.1073/pnas.1424440112 